\title{
IMPACT-INDUCED TENSILE WAVES IN A RUBBERLIKE MATERIAL*
}

\author{
JAMES K. KNOWLES ${ }^{\dagger}$
}

\begin{abstract}
This paper concerns the propagation of impact-generated tensile waves in a onedimensional bar made of a rubberlike material. Because the stress-strain curve changes from concave to convex as the strain increases, the governing quasi-linear system of partial differential equations, though hyperbolic, fails to be "genuinely nonlinear" so that the standard form of the boundary-initial value problem corresponding to impact is not well-posed at all levels of loading. When the problem fails to be well-posed, it does so by exhibiting a massive loss of uniqueness, even though an entropylike dissipation inequality is in force. Because the breakdown in uniqueness is reminiscent of a similar phenomenon that occurs in continuum-mechanical models for impact-induced phase transitions, a mathematically suitable, though physically unmotivated, supplementary selection mechanism for determining the solution naturally suggests itself. We describe in detail the solutions determined by two special forms of this selection mechanism, and we show that these two solutions provide bounds on the impact response, regardless of the selection principle used.
\end{abstract}

Key words. rubber, impact, genuine nonlinearity, kinetic relations

AMS subject classifications. 74B20, 74J30, 74J40, 74N20

PII. S0036139901388234

1. Introduction. For a thin bar or rod made of rubber and placed in an equilibrium state of uniaxial tension, the nominal stress, i.e., the force per unit undeformed cross-sectional area, is a monotonically increasing function of the longitudinal strain that is concave for small to moderate strains but strongly convex for large strains. This result was established experimentally by Treloar [1] in 1944; see Figure 5.4 in [2] or Figure 7.4(a) in [3]. Roughly speaking, the concave regime may be described by modeling the constituent polymer network with Gaussian statistics, while the strongly convex portion of the response arises from non-Gaussian effects; see Chapters 4-6, especially Figures 5.4 and 6.12 , of [2]. The present study is directed toward the effect of this change of curvature in the stress-strain curve upon the propagation of waves arising from tensile impact loading in a model that mimics rubber qualitatively.

For present purposes and in the one-dimensional setting to be described below, impact loading is modeled by specifying a particle velocity that is suddenly applied and maintained at the end of the bar, which is initially at rest in a stress-free configuration. The bar is taken to be semi-infinite to avoid reflections, which are unimportant as regards the issue of interest here. For a bar whose stress-strain relation is strictly concave for all strains, sudden tensile impact results in a "fan" or "rarefaction wave," while such impact produces a shock wave in a specimen whose stress-strain curve is strictly convex. The system of conservation laws governing the dynamics of a nonlinearly elastic bar in either of these two cases is not only hyperbolic but also genuinely nonlinear in terminology introduced by Lax [4] and described in the present context below; see also Chapter 11 of [5]. In the case of a rubberlike material, the basic system remains hyperbolic, but genuine nonlinearity fails. It is known that such a failure may result in a lack of uniqueness, even when an entropy-like inequality is

\footnotetext{
* Received by the editors April 18, 2001; accepted for publication (in revised form) October 21, 2001; published electronically April 3, 2002.

http://www.siam.org/journals/siap/62-4/38823.html

${ }^{\dagger}$ Division of Engineering and Applied Science, California Institute of Technology, Pasadena, CA 91125 (knowles@its.caltech.edu).
} 
in force; see [6]. For the rubberlike model studied here, there are three regimes of response, depending on the intensity of the loading. For weak impacts, corresponding to small impactor speeds, the response is a pure fan, as in a material with a concave stress-strain curve. For the severest impacts, the response is a pure shock wave, as in materials with convex stress-strain relations. For the intermediate range of impactor velocity, the response typically exhibits a "two-wave" structure consisting of a fan followed by a shock wave whose velocity is left undetermined in the conventional formulation of the problem. Thus, in this intermediate case, there is a one-parameter family of solutions to the boundary-initial value problem, each of which respects the dissipation inequality that plays the role of the second law of thermodynamics in the purely mechanical theory of one-dimensional nonlinear elasticity to be used here.

Two-wave response involving a moving strain discontinuity whose velocity fails to be determined by the boundary-initial value problem and the dissipation inequality also arises in the continuum-mechanical theory of solids such as shape-memory alloys or ceramics that are capable of undergoing impact-induced phase transitions [7]. Indeed, the underlying quasi-linear system of partial differential equations describing phase-transforming elastic solids is the same as the one to be investigated here, except that the stress-strain relation entering the theory in the case of a phase-transforming solid not only changes curvature but loses monotonicity as well. Thus while, for the rubberlike material, the system is always hyperbolic, that for the phase-transforming material undergoes a change of type from hyperbolic to elliptic over a portion of the range of strain.

In the impact problem for the phase-changing solid, there is a moving strain discontinuity - the phase boundary - separating the two material phases. The underlying physics of the phase transition provides the additional information needed to overcome the loss of uniqueness: One specifies continuum-mechanical versions of a nucleation criterion governing the initiation of the transition and of a kinetic relation relating the propagation speed of the phase boundary to the states on either side of it through the so-called driving force acting on the phase boundary. This remedy for lost uniqueness in the phase-transition problem suggests a way to resolve the corresponding mathematical issue for the rubberlike material considered here: One imposes the formalism of the kinetic relation to determine the unknown shock wave velocity; the analogue of the nucleation criterion turns out not to be needed. While this procedure renders the solution to the impact problem fully determined, it leaves unanswered the underlying physical question for rubber: What evolutionary process at the molecular level must be modeled to provide the necessary kinetic relation?

The role of a kinetic relation in strictly hyperbolic systems for which genuine nonlinearity fails has been discussed from the mathematical and computational points of view by LeFloch in the survey [6], which contains an extensive list of references to related work.

In the next section, we state the boundary-initial value problem modeling tensile impact in the rubberlike bar, and we describe the physically appropriate notion of dissipation and the associated "entropy inequality." In section 3, we elucidate the structure of solutions in each of the three regimes of impactor velocity alluded to above. The formalism of kinetic relations is introduced in section 4, and explicit solutions of the impact problem in the intermediate regime are constructed for two special cases: dissipation-free kinetics and maximally dissipative kinetics. We also show that these special solutions can be used to construct upper and lower bounds on the response of the bar in the intermediate range of impactor velocities regardless of 
the form of the kinetic relation. Section 5 is devoted to some general comments and unresolved questions.

2. The boundary-initial value problem. In its stress-free reference state, the one-dimensional bar to be studied occupies the nonnegative $x$-axis. After impact, a particle located at $x$ in the reference state is carried to $x+u(x, t)$, where $u$ is the longitudinal displacement of the particle at time $t$. It is assumed that $u$ is continuous with piecewise continuous first and second derivatives. The strain $\gamma(x, t)$ and particle velocity $v(x, t)$ are defined by $\gamma=u_{x}$ and $v=u_{t}$, respectively, where subscripts indicate partial derivatives. To ensure that the mapping $x \rightarrow x+u(x, t)$ is one-toone, it is assumed that $\gamma>-1$. The nominal stress at time $t$ at this particle is denoted by $\sigma(x, t)$. In a Lagrangian description of the motion, balance of linear momentum and kinematic compatibility are equivalent to the partial differential equations

$$
\sigma_{x}=\rho v_{t}, \quad v_{x}=\gamma_{t},
$$

where $\gamma$ and $v$ are smooth, and the jump conditions

$$
\llbracket \gamma \rrbracket \dot{s}+\llbracket v \rrbracket=0, \quad \llbracket \sigma \rrbracket+\rho \dot{s} \llbracket v \rrbracket=0
$$

at a moving strain discontinuity whose referential location is $x=s(t)$ at time $t$. Here $\llbracket g \rrbracket=g(s(t)+, t)-g(s(t)-, t)$. In (1) and $(2), \rho$ is the constant mass per unit referential volume, and $\dot{s}$ is the Lagrangian (or referential) velocity of the discontinuity.

The material of the bar is taken to be elastic so that

$$
\sigma=\hat{\sigma}(\gamma) \text { for } \gamma>-1
$$

where the stress-response function $\hat{\sigma}$ is given and assumed twice continuously differentiable. Note that the theory is purely mechanical rather than thermomechanical; thermal effects are omitted. For the material characterized by (3), the field equations (1) yield the basic partial differential equations

$$
\hat{\sigma}^{\prime}(\gamma) \gamma_{x}=\rho v_{t}, \quad v_{x}=\gamma_{t},
$$

where the prime means derivative. The system (4) is hyperbolic if $\hat{\sigma}^{\prime}(\gamma)>0$ for all $\gamma>-1$; it is genuinely nonlinear if it is hyperbolic and $\hat{\sigma}^{\prime \prime}(\gamma) \neq 0$ for all $\gamma>-1$. Hyperbolicity is assumed here, but genuine nonlinearity is not.

For many metals in tension $(\gamma>0)$, stress is a concave increasing function of strain, so that, to the extent that elasticity serves as an appropriate model, $\hat{\sigma}$ is concave. For vulcanized rubber, on the other hand, $\hat{\sigma}(\gamma)$ is concave only for strains of the order of 200 percent; for strains beyond this range, $\hat{\sigma}(\gamma)$ is strongly convex; see Figure 7.4(a) in [3] for experimental data confirming this assertion. To mimic this behavior, we shall consider the stress response function given by

$$
\sigma=\hat{\sigma}(\gamma)=E\left(\gamma-\gamma_{0} \gamma^{2}+\gamma^{3} / 3\right),
$$

where $E$ is Young's modulus for infinitesimal strains and $\gamma_{0}$ is a constant, $0<\gamma_{0}<1$. This $\hat{\sigma}(\gamma)$ is concave for $-1<\gamma<\gamma_{0}$ and convex for $\gamma>\gamma_{0}$. Note that (5) is not proposed here as a quantitatively valid model for rubber in uniaxial tension; for a discussion of a quantitatively appropriate constitutive law for rubber subject to deformations that include, but are not limited to, uniaxial stress, see Chapter 7 of [3]. 
Suppose that the bar governed by (3) is stretched to a strain $\gamma$; small-amplitude waves superposed on this state of strain will travel with the sound-wave speed $c$, where

$$
c=c(\gamma)=\sqrt{\hat{\sigma}^{\prime}(\gamma) / \rho}
$$

in particular, the speed of small amplitude waves at the undeformed state is

$$
c_{0}=c(0)=\sqrt{E / \rho} .
$$

For the special material (5), $c$ is given by

$$
c(\gamma)=c_{0} \sqrt{1-2 \gamma_{0} \gamma+\gamma^{2}}=c_{0} \sqrt{1-\gamma_{0}^{2}+\left(\gamma-\gamma_{0}\right)^{2}} ;
$$

note that this special $c(\gamma)$ has its minimum value $c_{m}$ at $\gamma=\gamma_{0}$, with

$$
c_{m}=\sqrt{1-\gamma_{0}^{2}} c_{0} .
$$

The graph of $c(\gamma)$ is shown in Figure 1.

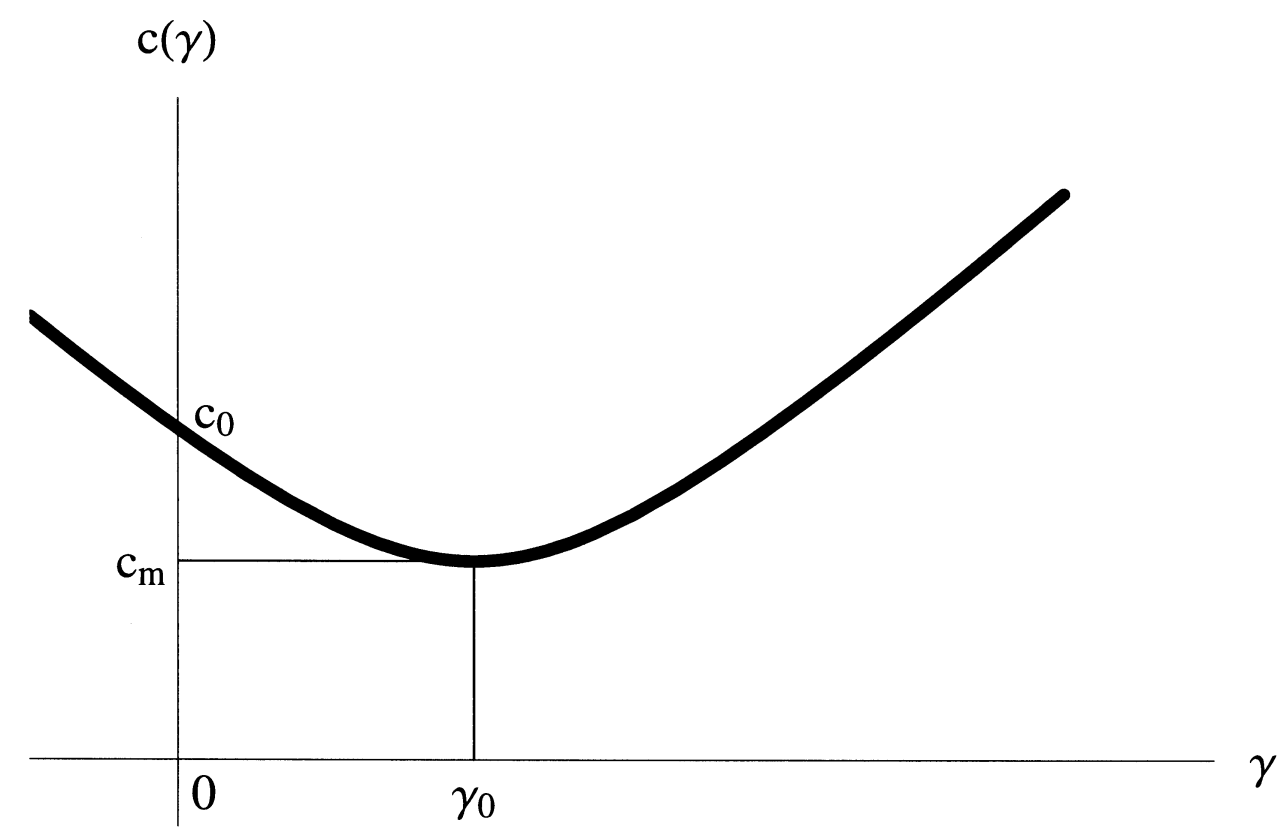

FIG. 1. The speed $c(\gamma)$ of small-amplitude waves superposed on the bar stretched to the strain $\gamma$ as a function of $\gamma$ for the material whose stress-response function is (5).

In the impact problem, the bar is assumed to be initially at rest in its undeformed state. At time $t=0$, a particle velocity $v=-V$ is imposed at the end $x=0$ and maintained for all subsequent time. Thus we have the following auxiliary conditions to accompany (4):

$$
\begin{gathered}
\gamma(x, 0)=0, \quad v(x, 0)=0 \text { for } x>0 \\
v(0, t)=-V \text { for } t>0
\end{gathered}
$$


We seek the solution of the impact problem (4), (10), (11) on the first quadrant of the $x, t$-plane.

Though the term "impact" might better be reserved for the case of compressive loading, for which $V$ in (11) is negative, we shall use the term for tensile loading as well; indeed, we shall consider only tensile impact so that we assume throughout that $V>0$. It may be remarked that tensile impact loading is achievable experimentally; see, for example, the early work of von Kármán and Duwez [8] on copper wires.

Because the impact problem just formulated does not involve a parameter with the dimension of either length or time, it is invariant under a change of scale $x \rightarrow$ $k x, t \rightarrow k t$. We consider only solutions that also have this invariance; these are necessarily functions of $\xi=x / t$ only. For such a scale-invariant solution, the first quadrant of the $x, t$-plane is divided into wedges issuing from the origin, on each of which the solution is either constant or takes the form of a fan.

The dissipation associated with the shock waves to be encountered below plays a significant role. Let $\gamma, v$ be a solution of the partial differential equations (4) that is smooth on a subinterval $\left[x_{1}, x_{2}\right]$ of the positive $x$-axis except at a moving discontinuity in $\gamma$ and $v$ that is located at $x=s(t)$ at time $t$. Let $D(t)$ be the rate of dissipation at time $t$, defined as the excess of the rate of work of the stresses acting at the ends of the portion of the bar under consideration over the rate of increase of total mechanical energy in that portion, all measured per unit referential cross-sectional area of the bar. Thus

$$
D(t)=\left.\sigma(x, t) v(x, t)\right|_{x_{1}} ^{x_{2}}-\frac{d}{d t} \int_{x_{1}}^{x_{2}}\left\{\frac{\rho}{2} v^{2}(x, t)+W(\gamma(x, t))\right\} d x,
$$

where

$$
W(\gamma)=\int_{0}^{\gamma} \hat{\sigma}\left(\gamma^{\prime}\right) d \gamma^{\prime}
$$

is the stored elastic energy per unit reference volume at the strain $\gamma$. With the help of (4) and (2), one can find the following alternate representation for $D(t)$ :

$$
D(t)=f(t) \dot{s}(t)
$$

where $f(t)$ is the driving force per unit cross-sectional area acting at time $t$ on the moving strain discontinuity; it is defined in terms of the strains $\gamma^{ \pm}(t)=\gamma(s(t) \pm, t)$ on either side of the jump by

$$
f=\hat{f}\left(\gamma^{+}, \gamma^{-}\right)=\int_{\gamma^{-}}^{\gamma^{+}} \hat{\sigma}(\gamma) d \gamma-\frac{\hat{\sigma}\left(\gamma^{+}\right)+\hat{\sigma}\left(\gamma^{-}\right)}{2}\left(\gamma^{+}-\gamma^{-}\right)
$$

Thus the rate of dissipation $D$ may be viewed as the rate of work done by the driving force $f$ in moving the discontinuity at the velocity $\dot{s}$. Note that $f$ may be interpreted geometrically as the signed area below the stress-strain curve and above its chord between the points $\left(\gamma^{-}, \hat{\sigma}\left(\gamma^{-}\right)\right)$and $\left(\gamma^{+}, \hat{\sigma}\left(\gamma^{+}\right)\right)$. When specialized from the present dynamical context to the setting of mechanical equilibrium, this notion of driving force is directly related to the concept of thermodynamic driving force that plays a major role in materials science in connection with phase transformations; see section 1.9 of [9]. 
A pair $\gamma, v$ satisfying the partial differential equations (4) and the jump conditions (2) is a physically admissible solution if it fulfills the following requirement at every strain discontinuity:

$$
D(t)=f(t) \dot{s}(t) \geq 0 \text { for } t>0,
$$

so that the total energy does not increase faster than the rate at which work is done. We adopt (16) as a postulate.

The driving force as defined in (15) has a number of important properties that lie near at hand. First, if there is in fact no discontinuity so that $\gamma^{+}=\gamma^{-}$, then $f$ vanishes, there is no dissipation, and (16) holds trivially. So if confined to smooth solutions of (4), nonlinear elasticity is a conservative theory. Second, if the material is linear so that $\hat{\sigma}(\gamma)=E \gamma$, the geometric interpretation of driving force shows that $f$ again vanishes so that linear elasticity is a conservative theory even if there are propagating strain discontinuities.

For a genuinely nonlinear material, it is clear geometrically that $f$ can never vanish if $\gamma^{+} \neq \gamma^{-}$. In particular, in an elastic material with a strictly concave stressstrain curve, a strain jump with a tensile back-state $\left(\gamma^{-}>0\right)$ moving with positive speed $\dot{s}$ into unstrained material $\left(\gamma^{+}=0\right)$ will violate the dissipation inequality since $f<0$ in this case. Thus tensile shock waves cannot propagate into undisturbed material when $\hat{\sigma}(\gamma)$ is strictly concave, as it is for many metals.

For the rubberlike material characterized by (5), direct calculation shows that $f$ is given by

$$
f=\frac{E}{12}\left(\gamma^{+}-\gamma^{-}\right)^{3}\left(2 \gamma_{0}-\gamma^{+}-\gamma^{-}\right) .
$$

In this case, a solution $(\gamma, v)$ may possess genuine discontinuities for which $f$ vanishes; such discontinuities must satisfy $\gamma^{+}+\gamma^{-}=2 \gamma_{0}$, as is clear from (17) or from the antisymmetry of the stress-strain curve about its point of inflection at $\gamma=\gamma_{0}$.

The definition (15) also applies for the systems of mixed type that arise in continuum-mechanical modeling of phase transitions in elastic solids, where it plays a major role in the formulation of nucleation criteria and kinetic relations controlling the initiation and evolution of such transitions; see [7]. There is a generalization of (15) that takes thermal effects into account [10].

\section{Solutions in the three regimes of impactor velocity.}

3.1. Weak impacts. We first examine the case in which the impactor speed $V$ is small enough to keep the resulting maximum strain below the level $\gamma=\gamma_{0}$. The fact that a tensile shock wave cannot propagate into undisturbed material when $\hat{\sigma}(\gamma)$ is strictly concave suggests that a fan will arise in this case, providing a solution $\gamma, v$ that is continuous, and therefore dissipation-free, on the first quadrant of the $x, t$-plane. We set $\xi=x / t$ and look for a solution in the form

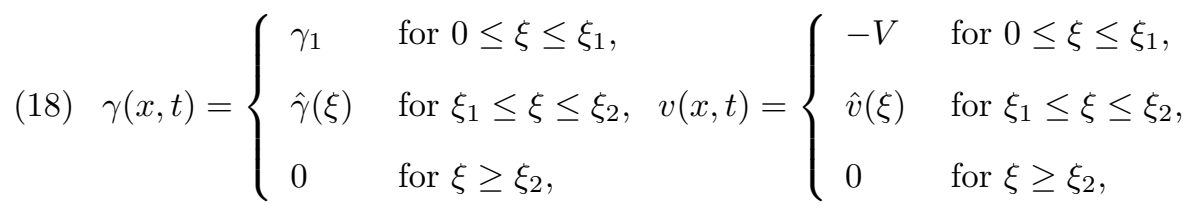




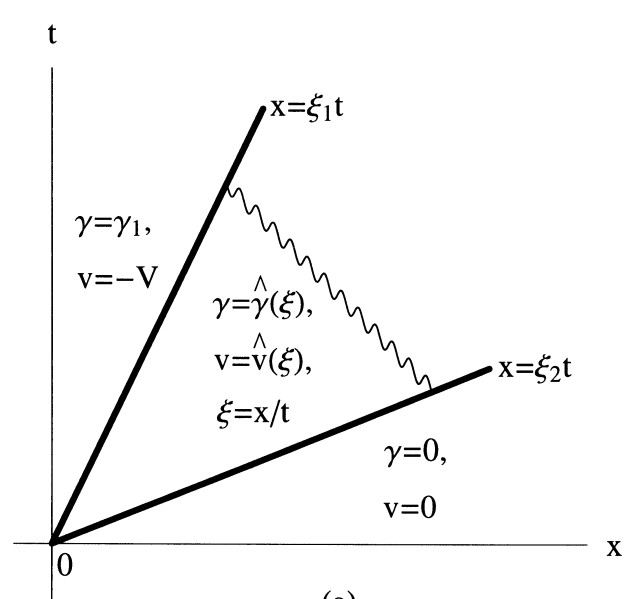

(a)

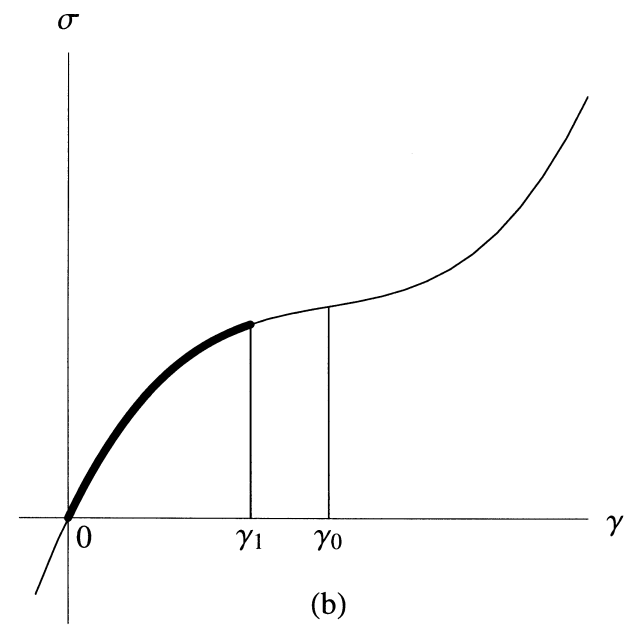

FiG. 2. Solution in the form of a fan for the case of weak impact. (a) The x,t-plane. (b) The portion of the stress-strain curve participating in the fan (shown in bold).

where $\gamma_{1}=\hat{\gamma}\left(\xi_{1}\right)$ and $\xi_{1}, \xi_{2}$ are to be determined; see Figure 2. Substituting from (18) into (4) and requiring that $\hat{\gamma}$ and $\hat{v}$ not be identically constant lead to the conclusion that $\hat{\gamma}$ is to be found from

$$
c(\hat{\gamma}(\xi))=\xi,
$$

where $c(\gamma)$ is the sound-wave speed defined in (6). Continuity and the initial conditions (10) require that $\hat{\gamma}(\xi) \rightarrow 0$ as $\xi \rightarrow \xi_{2}-$ so that in fact $\xi_{2}=c_{0}$. Bearing Figure 1 in mind, one notes that the values of $\hat{\gamma}(\xi)$ must all lie in the interval $\left[0, \gamma_{0}\right] ;(19)$ then determines $\hat{\gamma}(\xi)$ as a single-valued, montonically decreasing function of $\xi$. Having $\hat{\gamma}(\xi)$, the particle velocity $\hat{v}(\xi)$ in the fan is determined from (4), which becomes

$$
\hat{v}^{\prime}(\xi)=-\xi \hat{\gamma}^{\prime}(\xi) ;
$$

(20) and the vanishing of $\hat{v}(\xi)$ at $\xi=\xi_{2}=c_{0}$ furnishes

$$
\hat{v}(\xi)=\int_{\xi}^{c_{0}} \zeta \hat{\gamma}^{\prime}(\zeta) d \zeta .
$$

The further continuity requirement $\hat{v}\left(\xi_{1}\right)=-V$ yields

$$
\int_{\xi_{1}}^{c_{0}} \xi \hat{\gamma}^{\prime}(\xi) d \xi=-V .
$$

The change of variables $\xi \rightarrow \gamma=\hat{\gamma}(\xi)$ converts (22) to

$$
\int_{0}^{\gamma_{1}} c(\gamma) d \gamma=V,
$$

from which $\gamma_{1}=\hat{\gamma}\left(\xi_{1}\right)$ is to be determined. Once $\gamma_{1}$ is known, $\xi_{1}$ is found from (19).

Since $\xi_{1}<c_{0}$, one has $\gamma_{1}=\hat{\gamma}\left(\xi_{1}\right)>0$ so that (23) has no solution if $V<0$, i.e., if the impact is compressive. When $V>0$, the maximum strain in the fan is the value 


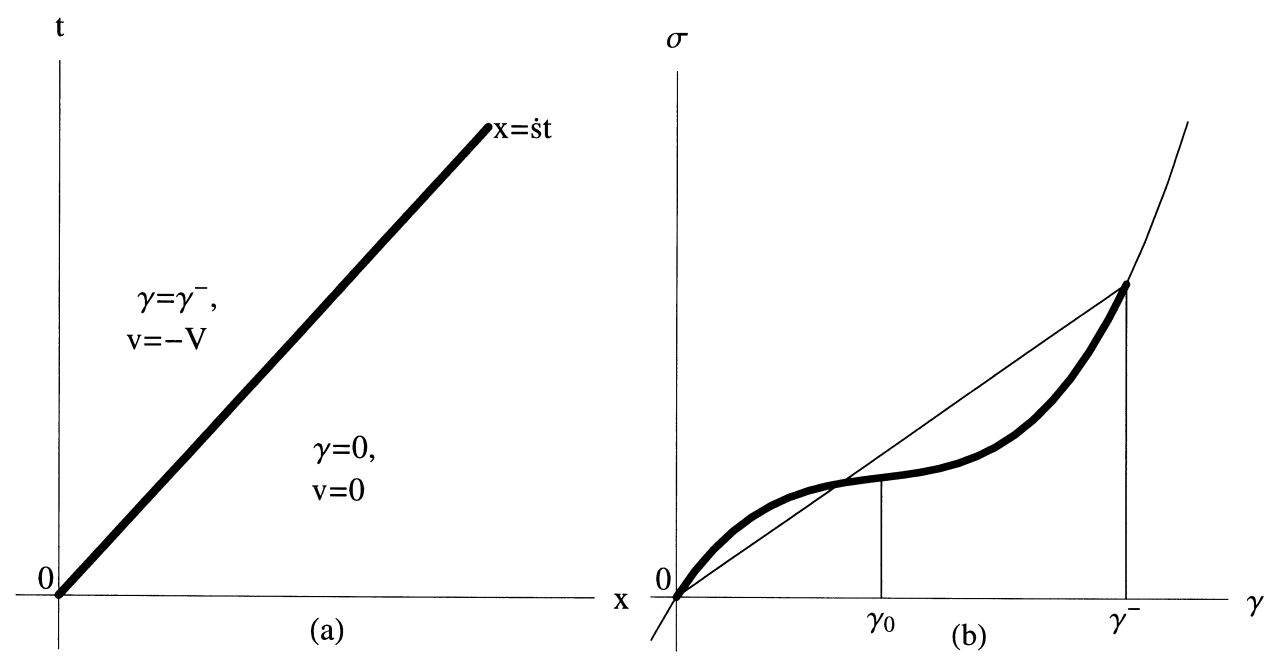

FIG. 3. Solution in the form of a shock wave for the case of strong impact. (a) The $x, t$-plane. (b) The chord connecting the undeformed state and the back-state on the stress-strain curve.

$\gamma_{1}$ found in terms of $V$ from (23). To keep $\gamma_{1} \leq \gamma_{0}$, the impactor velocity $V$ must satisfy $V \leq V_{\star}$, where

$$
V_{\star}=\int_{0}^{\gamma_{0}} c(\gamma) d \gamma
$$

Thus the restriction

$$
0<V \leq V_{\star} \quad \text { (weak impact) }
$$

on the impactor velocity delineates the regime of weak impacts.

Using (8) in (19), one finds the strain in the fan explicitly as

$$
\gamma(x, t)=\hat{\gamma}(x / t)=\gamma_{0}-\left(1 / c_{0}\right) \sqrt{x^{2} / t^{2}-c_{m}^{2}} \text { for } \xi_{1} t \leq x \leq c_{0} t
$$

where $c_{m}$ is given by $(9)$; the critical impactor velocity $V_{\star}$ is

$$
V_{\star}=\left\{\frac{\gamma_{0}}{2}+\frac{1-\gamma_{0}^{2}}{4} \log \left(\frac{1+\gamma_{0}}{1-\gamma_{0}}\right)\right\} c_{0} .
$$

One can show that $\hat{\gamma}^{\prime \prime}(\xi)>0$ so that the strain is a convex function of $\xi$ in the fan. A schematic diagram of the spatial strain distribution at a fixed time $t$ arising from a weak impact is shown in part (a) of Figure 8 below.

3.2. Strong impacts. Next we consider the case of large impactor speeds $V$, postponing the more complex case in which $V$ is in an intermediate range. Since one might now expect behavior similar to that of a material with a convex stress-strain curve, it is natural to seek a solution with a shock wave, with the bar jumping directly from the unstressed state to a severely strained configuration behind the shock; see Figure 3. Thus we try

$$
\gamma(x, t)=\left\{\begin{array}{ll}
\gamma^{-} & \text {for } 0 \leq x<\dot{s} t, \\
0 & \text { for } x>\dot{s} t,
\end{array} \quad v(x, t)= \begin{cases}-V & \text { for } 0 \leq x<\dot{s} t, \\
0 & \text { for } x>\dot{s} t\end{cases}\right.
$$


where the back-state strain $\gamma^{-}$and the constant Lagrangian shock velocity $\dot{s}$ are to be determined.

Since $\gamma^{-}$and $V$ are constants, the differential equations (4) are trivially satisfied; only the jump conditions remain. These specialize to

$$
-\gamma^{-} \dot{s}+V=0, \quad-\hat{\sigma}\left(\gamma^{-}\right)+\rho \dot{s} V=0 .
$$

Eliminating $\dot{s}$ from these yields the equation to determine $\gamma^{-}$:

$$
\gamma^{-} \hat{\sigma}\left(\gamma^{-}\right)=\rho V^{2}
$$

for the special material (5) this becomes

$$
\gamma^{-} \sqrt{1-\gamma_{0} \gamma^{-}+\left(\gamma^{-}\right)^{2} / 3}=V / c_{0} .
$$

Once $\gamma^{-}$is determined in terms of $V$, the shock speed is found from

$$
\dot{s}=V / \gamma^{-} .
$$

Since the strain $\gamma^{+}$ahead of the shock wave vanishes, the driving force (17) is given by

$$
f=\frac{E}{12}\left(\gamma^{-}\right)^{3}\left(\gamma^{-}-2 \gamma_{0}\right)
$$

Because $\dot{s}>0$, the dissipation inequality (16) requires that $f \geq 0$ and hence that

$$
\gamma^{-} \geq 2 \gamma_{0} \text { (physical admissibility). }
$$

The left side of (31) is a monotonically increasing function of $\gamma^{-}$for $\gamma^{-} \geq 2 \gamma_{0}$ that takes all values at least as great as $2 \gamma_{0} \sqrt{1-2 \gamma_{0}^{2} / 3}$. It follows that (31) has a unique solution $\gamma^{-}$corresponding to a physically admissible shock wave, provided $V>V_{\star \star}$, where

$$
V_{\star \star}=2 \gamma_{0} \sqrt{1-2 \gamma_{0}^{2} / 3} c_{0} .
$$

From (27) and (35), one can show that $V_{\star \star}>V_{\star}$ for all $\gamma_{0}$ in $(0,1)$ so that there is an interval $V_{\star}<V<V_{\star \star}$, where neither the pure-fan solution nor the pure-shock-wave solution applies.

As the impactor velocity $V$ increases, the back-state strain $\gamma^{-}$as determined from (31) also increases; the shock speed $\dot{s}$ is also an increasing function of $V$.

It is easily verified from (31) and (32) that when the back-state strain takes the value $\gamma^{-}=3 \gamma_{0}$, the Lagrangian shock-wave speed and the impactor velocity take the respective values $\dot{s}=c_{0}$ and $V=V_{\star \star \star}$, where

$$
V_{\star \star \star}=3 \gamma_{0} c_{0} .
$$

Thus in those pure-shock-wave solutions just constructed for which $\gamma^{-}>3 \gamma_{0}$ and therefore $V>V_{\star \star \star}$, the shock wave is supersonic (in the Lagrangian sense) with respect to the undisturbed state ahead of it. This property, which will assume further significance in the next subsection, leads us to define the regime of strong impact as that corresponding to impactor velocities for which

$$
V>V_{\star \star \star} \quad \text { (strong impact). }
$$




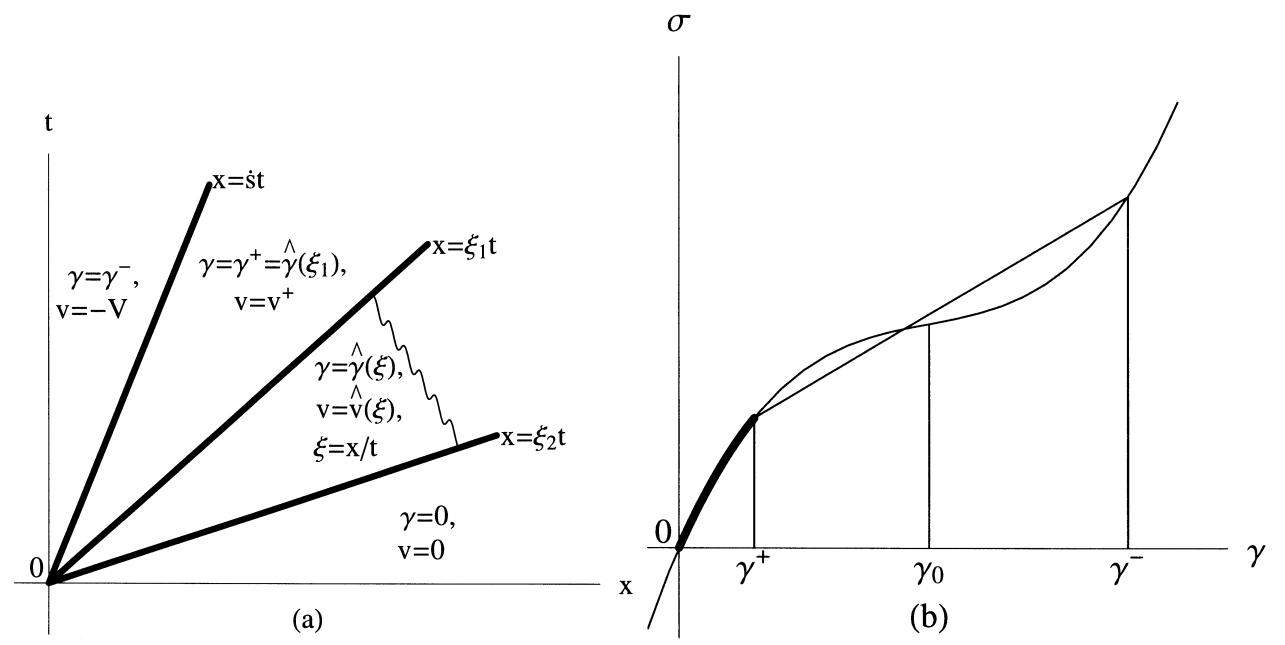

FIG. 4. Solution with a fan and a shock wave for the case of impacts of intermediate strength. (a) The $x$,t-plane. (b) The portion of the stress-strain curve participating in the fan (shown in bold) and the chord connecting the states on either side of the shock wave.

The spatial distribution of strain for a strong impact is as shown schematically in Figure 8(d) below.

3.3. Impacts of intermediate strength. With the hope of filling the gap $V_{\star}<V<V_{\star \star}$, where as yet there is no solution, we now attempt to construct one that involves both a fan and a shock wave, as in Figure 4. Thus we now propose that $\gamma$ and $v$ have the following two-wave form:

$$
\gamma(x, t)=\left\{\begin{array}{ll}
\gamma^{-} & \text {for } 0 \leq x<\dot{s} t \\
\gamma^{+} & \text {for } \dot{s} t<x \leq \xi_{1} t, \\
\hat{\gamma}(x / t) & \text { for } \xi_{1} t \leq x \leq \xi_{2} t, \\
0 & \text { for } x \geq \xi_{2} t
\end{array} \quad v(x, t)= \begin{cases}-V & \text { for } 0 \leq x<\dot{s} t \\
v^{+} & \text {for } \dot{s} t<x \leq \xi_{1} t \\
\hat{v}(x / t) & \text { for } \xi_{1} \leq x \leq \xi_{2} t \\
0 & \text { for } x \geq \xi_{2} t\end{cases}\right.
$$

where $\gamma^{-}, \dot{s}, \xi_{1}, \xi_{2}, \hat{\gamma}(\xi)$, and $\hat{v}(\xi)$ are to be determined and, by continuity, $\gamma^{+}=\hat{\gamma}\left(\xi_{1}\right)$, $v^{+}=\hat{v}\left(\xi_{1}\right)$.

As in the fan arising from weak impacts, one has $\xi_{2}=c_{0}$; since the shock wave is to trail the fan, we require that $\dot{s} \leq \xi_{1}$. Assuming that $\gamma$ and $v$ are continuous everywhere except across the shock, one has $\xi_{1}=c\left(\gamma^{+}\right)$so that

$$
\dot{s} \leq c\left(\gamma^{+}\right) .
$$

The fan equations (19), (20) hold in the present case as well. Since $\hat{v}\left(\xi_{2}\right)=0$, one finds from (20), (19) that

$$
v^{+}=\hat{v}\left(\xi_{1}\right)=\int_{\xi_{1}}^{c_{0}} \xi \hat{\gamma}^{\prime}(\xi) d \xi=-\int_{0}^{\gamma^{+}} c(\gamma) d \gamma
$$


Using (40) in the jump conditions (2) applied at the shock $x=\dot{s} t$ yields

$$
\begin{gathered}
\left(\gamma^{+}-\gamma^{-}\right) \dot{s}-\left\{\int_{0}^{\gamma^{+}} c(\gamma) d \gamma-V\right\}=0, \\
\hat{\sigma}\left(\gamma^{+}\right)-\hat{\sigma}\left(\gamma^{-}\right)-\rho \dot{s}\left\{\int_{0}^{\gamma^{+}} c(\gamma) d \gamma-V\right\}=0 .
\end{gathered}
$$

These are two equations for the front- and back-state strains $\gamma^{ \pm}$at the shock; they involve the shock speed $\dot{s}$ as an unknown parameter and the impactor velocity $V$ as a given datum.

Eliminating the common contents of the braces between (41) and (42) gives the standard formula relating the shock speed and the slope of the chord connecting the two points on the stress-strain curve that correspond to the front- and back-states of the shock:

$$
\rho \dot{s}^{2}=\frac{\hat{\sigma}\left(\gamma^{+}\right)-\hat{\sigma}\left(\gamma^{-}\right)}{\gamma^{+}-\gamma^{-}} .
$$

If $\gamma^{+} \neq \gamma^{-}$, the system (41), (42) is clearly equivalent to either (41) or (42), together with (43).

For the special material (5), (41) and (43) take the respective forms

$$
\begin{gathered}
\left(\gamma^{+}-\gamma^{-}\right) \dot{s}+c_{0} \int_{\gamma^{+}}^{\gamma_{0}} \sqrt{1-\gamma_{0}^{2}+\left(\gamma-\gamma_{0}\right)^{2}} d \gamma+w=0, \\
\left(\gamma^{+}-\gamma_{0}\right)^{2}+\left(\gamma^{+}-\gamma_{0}\right)\left(\gamma^{-}-\gamma_{0}\right)+\left(\gamma^{-}-\gamma_{0}\right)^{2}=3\left(\dot{s}^{2}-c_{m}^{2}\right) / c_{0}^{2},
\end{gathered}
$$

where in (44) we have introduced the departure $w$ of the impactor velocity $V$ from the critical velocity $V_{\star}$ delimiting the pure-fan, weak-impact regime,

$$
w=V-V_{\star},
$$

and we have used (24) in rewriting (41). In (45), $c_{m}$ and $c_{0}$ are the minimum sound speed and the sound speed at the undeformed state, respectively. We shall view (44) and (45) as the system replacing (41), (42).

The left side of (45) is nonnegative so that necessarily $\dot{s} \geq c_{m}$; together with (39), this yields

$$
c_{m} \leq \dot{s} \leq c\left(\gamma^{+}\right)
$$

Since $\dot{s}>0$, physical admissibility in the sense of (16) requires that the shock wave must bear a nonnegative driving force. Since in (17) one now has $\gamma^{-}>\gamma^{+}$, this implies that

$$
\gamma^{+}+\gamma^{-} \geq 2 \gamma_{0}
$$

If in (47) one represents $\dot{s}$ through (45) and $c\left(\gamma^{+}\right)$through (8), one finds, after simplification, the further restriction

$$
2 \gamma^{+}+\gamma^{-} \leq 3 \gamma_{0}
$$




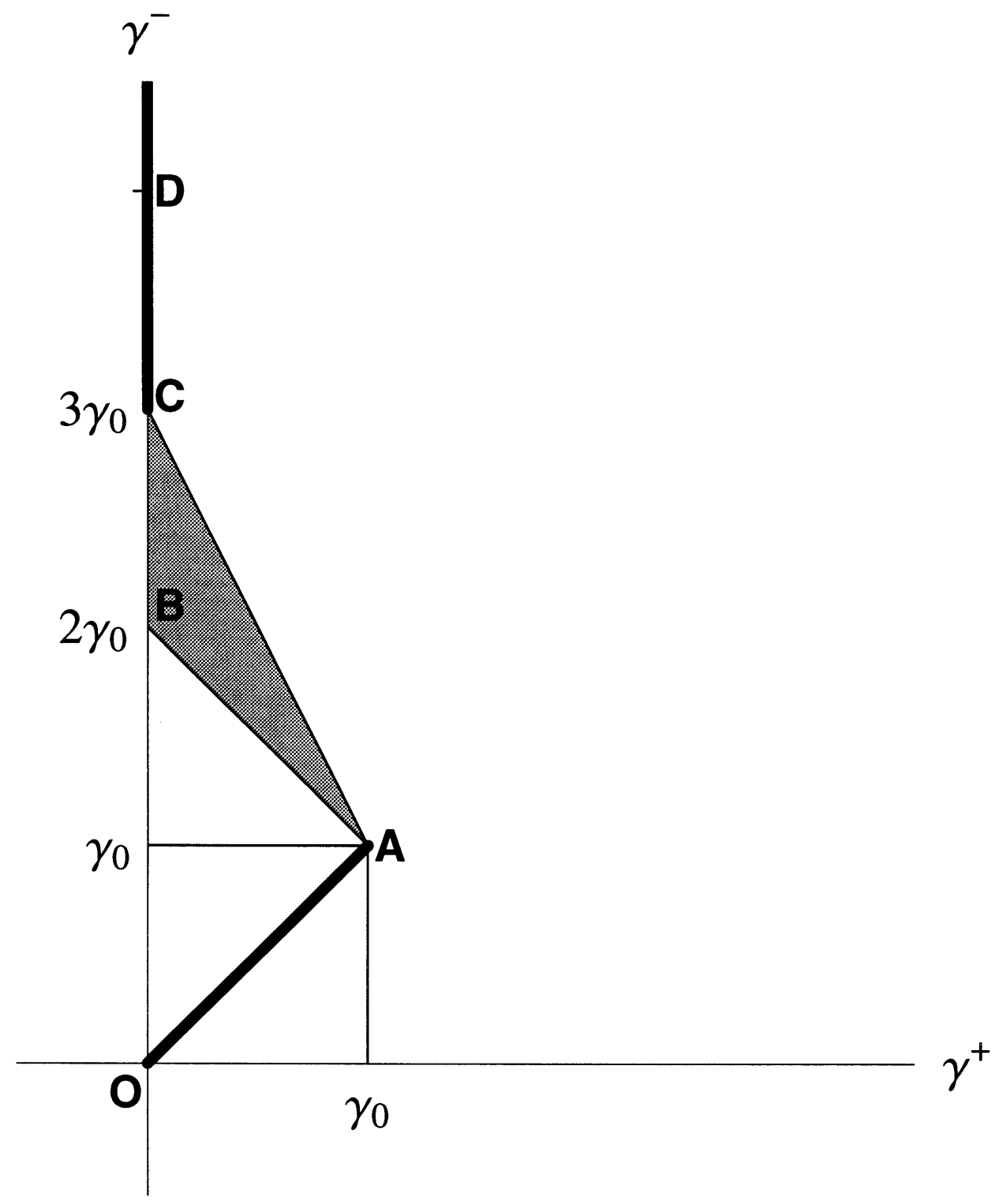

FIG. 5. Pairs $\left(\gamma^{+}, \gamma^{-}\right)$satisfying (41), (42) must correspond to points in the shaded triangle ABC in the $\gamma^{+}, \gamma^{-}$-plane. Points of the form $\left(0, \gamma^{-}\right)$with $\gamma^{-} \geq 2 \gamma_{0}$, lying on the vertical axis, correspond to the pure-shock-wave solutions of subsection 3.2. Points on the bold line $\gamma^{+}=\gamma^{-}$with $0<\gamma^{+}<\gamma_{0}$ correspond to the pure fan of subsection 3.1 .

Pairs $\left(\gamma^{+}, \gamma^{-}\right)$satisfying the inequalities (48), (49) as well as $\gamma^{+} \leq \gamma_{0}$ correspond to points in the shaded triangle $\mathbf{A B C}$ in the $\gamma^{+}, \gamma^{-}$-plane of Figure 5.

The pure-shock-wave solutions of subsection 3.2 may also be represented in the $\gamma^{+}, \gamma^{-}$-plane of Figure 5; since in these one has $\gamma^{+}=0, \gamma^{-} \geq 2 \gamma_{0}$, they correspond to the portion of the vertical axis above the point $\mathbf{B}$ in the figure. Those pure-shock-wave solutions corresponding to the segment $\mathbf{B C}$ of the $\gamma^{-}$-axis are actually limiting cases as $\gamma^{+} \rightarrow 0$ of the mixed fan-plus-shock solutions associated with points within the 
shaded triangle $\mathbf{A B C}$. The remaining shock-wave solutions - those associated with the portion (shown bold) of the $\gamma^{-}$-axis above the point $\mathbf{C}$ - are precisely those arising from strong impacts $\left(V>V_{\star \star \star \star}\right)$ whose shock-wave speeds $\dot{s}$ are supersonic with respect to their front-state; see the remarks at the end of the preceding subsection. Thus we speak of impacts of intermediate strength as those for which

$$
V_{\star}<V<V_{\star \star \star} \text { (intermediate impact). }
$$

The pure-fan solutions of subsection 3.1 correspond to the line segment (also shown bold) in the plane of Figure 5 for which $0<\gamma^{-}=\gamma^{+} \leq \gamma_{0}$.

Suppose that, for a given $w$ and a given $\dot{s} \geq c_{m}$, strains $\gamma^{+}<\gamma_{0}$ and $\gamma^{-}>\gamma_{0}$ satisfying (44), (45), and (47)-(49) have been determined. Since $\xi_{1}=c\left(\gamma^{+}\right)$and $\xi_{2}=c_{0}$, the speeds at the head and tail of the fan are known once $\gamma^{+}$and $\gamma^{-}$ have been found. Define $\hat{\gamma}(\xi)$ and $\hat{v}(\xi)$ by (26) and (21), respectively. Then $\gamma(x, t)$ and $v(x, t)$, when constructed from these ingredients according to (38), fulfill the differential equations (4), the jump conditions (2), the initial conditions (10), the boundary condition (11), and the physical admissibility condition (16) despite the fact that the shock-wave speed $\dot{s}$ has not been found. This suggests that (44) and (45) should determine a one-parameter family of admissible solutions (38) of the boundaryinitial value problem.

The shaded triangular region in Figure 5, along with the portions of straight lines that represent the pure-fan and pure-shock-wave solutions, may now be mapped through (44)-(46) into a plane in which the natural Cartesian coordinates are the dimensionless shock-wave velocity $\dot{s} / c_{m}$ and the dimensionless impactor velocity $V /\left(\sqrt{1-\gamma_{0}^{2}} c_{m}\right)$. Consider first the pure-fan solutions. Setting $\gamma^{-}=\gamma^{+}=\gamma_{0}-$ $\sqrt{1-\gamma_{0}^{2}} \eta$ in (44), (45) and using (46) lead to the parametric equations

$$
\left.\begin{array}{r}
\dot{s} / c_{m}=\sqrt{1+\eta^{2}}, \\
V /\left(\sqrt{1-\gamma_{0}^{2}} c_{m}\right)=H(h)-H(\eta)
\end{array}\right\} \text { for } 0 \leq \eta \leq h,
$$

where

$$
H(\eta)=\int_{0}^{\eta} \sqrt{1+\zeta^{2}} d \zeta \text { for } 0 \leq \eta \leq h \text { and } h=\gamma_{0} / \sqrt{1-\gamma_{0}^{2}} .
$$

If one views the pure-fan solution as a limiting case of the fan-plus-shock solution in which the shock has collapsed to the tail of the fan, one would have $\dot{s}=c(\gamma+)$ in this limit; this is the meaning to be attached to $\dot{s}$ on the curve in the $\dot{s}, V$-plane represented by (51).

Next we map the segment of the boundary of the triangle for which $\gamma^{-}=2 \gamma_{0}-\gamma^{+}$. From (44)-(46), one finds that the image of this segment in the dimensionless $\dot{s}, V$ plane is the curve represented parametrically by

$$
\left.\begin{array}{r}
\dot{s} / c_{m}=\sqrt{1+\eta^{2} / 3}, \\
V /\left(\sqrt{1-\gamma_{0}^{2}} c_{m}\right)=F(\eta)+H(h)
\end{array}\right\} \text { for } 0 \leq \eta \leq h,
$$

where

$$
F(\eta)=2 \eta \sqrt{1+\eta^{2} / 3}-H(\eta) \text { for } 0 \leq \eta \leq h .
$$




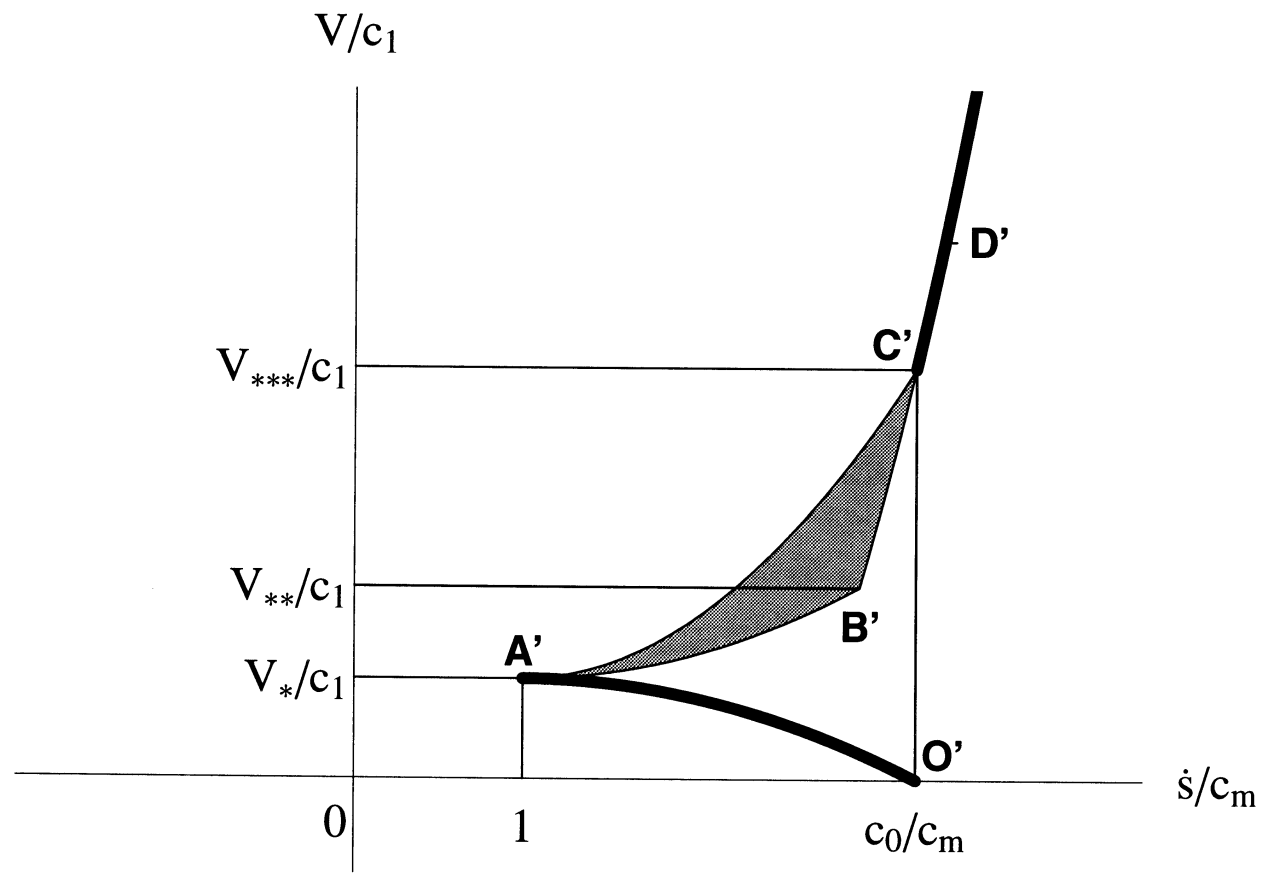

FIG. 6. Pairs $\left(\dot{s} / c_{m}, V / c_{1}\right)$ corresponding to points $\left(\gamma^{+}, \gamma^{-}\right)$in the locus of Figure 5 ; here we have written $c_{1}=\sqrt{1-\gamma_{0}^{2}} c_{m}$ for simplicity. Values of $\dot{s}$ and $V$ for which a mixed fan-shock-wave solution exists must correspond to points in the shaded region. The loci corresponding to pure-shock and pure-fan solutions are shown bold.

From (44)-(46), the image of the segment of the boundary of the triangle on which $\gamma^{-}=3 \gamma_{0}-2 \gamma^{+}$is found to be the curve represented by

$$
\left.\begin{array}{r}
\dot{s} / c_{m}=\sqrt{1+\eta^{2}}, \\
V /\left(\sqrt{1-\gamma_{0}^{2}} c_{m}\right)=G(\eta)+H(h)
\end{array}\right\} \text { for } 0 \leq \eta \leq h,
$$

where

$$
G(\eta)=3 \eta \sqrt{1+\eta^{2}}-H(\eta) \text { for } 0 \leq \eta \leq h .
$$

Finally, setting $\gamma^{+}=0, \gamma^{-}=\gamma_{0}+\sqrt{1-\gamma_{0}^{2}} \eta$ in (44), (45) furnishes a representation of the image in the dimensionless $\dot{s}, V$-plane of the segment of the vertical axis in the $\gamma^{+}, \gamma^{-}$-plane (Figure 5) that corresponds to the pure-shock-wave solutions:

$$
\left.\begin{array}{r}
\dot{s} / c_{m}=\sqrt{1+(1 / 3)\left(h^{2}-h \eta+\eta^{2}\right)}, \\
V /\left(\sqrt{1-\gamma_{0}^{2}} c_{m}\right)=(h+\eta) \sqrt{1+(1 / 3)\left(h^{2}-h \eta+\eta^{2}\right)}
\end{array}\right\} \quad \text { for } \eta \geq h .
$$

Figure 6 shows the image just constructed in the dimensionless $\dot{s}, V$-plane of the locus in the $\gamma^{+}, \gamma^{-}$-plane describing the various types of solutions as represented in Figure 5. It may be noted that the points on the curve (57) for which $\eta=h$ and $\eta=2 h$ coincide, respectively, with the end points $\mathbf{B}^{\prime}$ and $\mathbf{C}^{\prime}$ of the curves (53) and (55) forming part of the boundary of the region corresponding to the mixed fan-shock-wave solutions. 
If, for given $V$ and given $\dot{s}$, there is a physically admissible solution $\gamma^{+}, \gamma^{-}$of the mixed fan-shock equations (44), (45) with $0 \leq \gamma^{+} \leq \gamma_{0}$ and for which $c\left(\gamma^{+}\right) \geq \dot{s}$, then we have shown that the point corresponding to $\dot{s}, V$ must lie in the shaded region in Figure 6 . We have not proved the converse, which asserts that, for every pair $\dot{s}, V$ corresponding to a point in the shaded region of Figure 6, there is an acceptable solution $\gamma^{+}, \gamma^{-}$of (44), (45). Numerical experimentation with these equations, however, is consistent with this assertion, which we take for granted in what follows.

One can draw several immediate conclusions with the help of Figure 6, which summarizes all of the results obtained in the present section:

(i) For each impactor velocity $V$ between zero and $V_{\star}$, there is a unique pure-fan solution to the impact problem corresponding to the point on the curve $\mathbf{A}^{\prime} \mathbf{O}^{\prime}$ singled out by this value of $V$.

(ii) For each $V$ between $V_{\star}$ and $V_{\star \star \star}$, there is a one-parameter family of solutions corresponding to points along a horizontal line in the shaded region, each solution having a fan and a shock wave. In this regime, a mechanism is needed to specify the shock-wave velocity in order to select a particular solution from the one-parameter family. If $V_{\star \star}<V<V_{\star \star \star}$, the horizontal line terminates on the $\operatorname{arc} \mathbf{B}^{\prime} \mathbf{C}^{\prime}$, whose points represent pure-shock-wave solutions, the fan having disappeared as $\gamma^{+} \rightarrow 0+$.

(iii) In the regime of impactor velocities for which $V>V_{\star \star \star}$, each $V$ corresponds to a unique solution of the pure-shock-wave type represented by a point on the arc containing the points $\mathbf{C}^{\prime}$ and $\mathbf{D}^{\prime}$.

We turn in the next section to the questions surrounding the selection of solutions when $V_{\star}<V<V_{\star \star \star \star}$.

In the case of a Riemann problem for a first-order partial differential equation that represents a scalar conservation law for which genuine nonlinearity fails, LeFloch in section 3 of [6] constructs a solution involving both a shock wave and a fan. This is done by augmenting the conservation law with higher-order terms that correspond to small viscous and dispersive effects; this augmentation furnishes a selection mechanism. The augmented equation is solved numerically. Fan-plus-shock solutions to a Riemann problem for the elastodynamic system (4) are found numerically in section 5 (Figure 5.1) of [6].

A Riemann problem for the elastodynamic system (4) in which $\hat{\sigma}(\gamma)$ is cubic in $\gamma$ is considered by both Hayes and LeFloch [11] and Schulze and Shearer [12] for the case in which the system is hyperbolic but genuine nonlinearity fails. In [11], the authors find mixed fan-shock solutions using numerical schemes based on viscosity-plus-straingradient augmentation. In [12], a complete description of admissible solutions of the Riemann problem for the elastodynamic system is obtained with the help of travelling wave solutions of the augmented system; the methods are fully analytic. The authors note that the nucleation condition is not required when $\hat{\sigma}(\gamma)$ is monotonic.

\section{Kinetic relations and special solutions.}

4.1. Kinetic relations. In the continuum-mechanical theory of phase transitions in solids described in $[7,10]$, a kinetic relation is taken to be a relation between the driving force acting on the phase boundary and the phase boundary velocity:

$$
f=\phi(\dot{s}),
$$

where $\phi$ is a materially determined function that is required by the dissipation inequality (16) to satisfy

$$
\phi(\dot{s}) \dot{s} \geq 0
$$


The detailed form of $\phi$ appropriate to a particular phase transition in a crystal, for example, might be constructed either through modeling at the level of the atomic lattice, as in thermal activation theory [13], or through phenomenological considerations. A technique of the latter type, pioneered for phase transitions by Truskinovsky [14] and Slemrod [15], involves augmenting the constitutive law (3) by adding to the "elastic part" $\hat{\sigma}(\gamma)$ of the stress a viscous contribution as well as a strain-gradient term. In the limit as these extra terms vanish, traveling-wave solutions with diffuse phase boundaries in the augmented theory tend to solutions in the unaugmented theory that possess sharp phase boundaries propagating according to a special kinetic law of the form (58) inherited from - and depending upon - the particular augmentation used; see [16]. Such a limit process is exploited by Hayes and LeFloch [11] and by Schulze and Shearer [12] in their treatments of the elastodynamic Riemann problem in the absence of genuine nonlinearity. Augmentation of this kind, when physically valid, is useful computationally since it avoids the need to track or capture the sharp phase boundaries of the unaugmented theory.

In general, $\phi(\dot{s})$ is taken to be a monotonically increasing function of $\dot{s}$, though interesting phenomena such as stick-slip phase-boundary motion arise when this assumption is relinquished [17].

In [18], it is shown that a particular kinetic relation of the form (58) provides a quantitatively accurate description of the experimental results of Erskine and Nellis [19] concerning an impact-induced graphite-to-diamond phase transition.

Although there is no apparent physical reason to do so, we now explore the mathematical consequences of assuming that a relation of the form (58) holds in the present setting. We shall continue to use the term "kinetic relation" in referring to (58), despite the fact that we have not identified the underlying physical process whose kinetics it controls. To implement the assumption, we first note that the triangular region $\mathbf{A B C}$ in the $\gamma^{+}, \gamma^{-}$-plane of Figure 5 may be mapped to the $\dot{s}, f$-plane by means of (17) and (45); the resulting image is the curvilinear triangle $\mathbf{A}^{\prime \prime} \mathbf{B}^{\prime \prime} \mathbf{C}^{\prime \prime}$ shown shaded in the schematic Figure 7 . The curve in the $\dot{s}, f$-plane represented by (58) must lie in the shaded region, as indicated in the figure.

We now set out two examples in which particular kinetic relations of the form (58) are used as selection mechanisms to single out solutions of the impact problem in the regime where a one-parameter family of solutions is available.

4.2. Dissipation-free kinetics. In the first example, we take

$$
f=\phi(\dot{s}) \equiv 0 .
$$

According to (14), the response to impact is dissipation-free in this case. By (17), the choice (60) is equivalent to assuming that

$$
\gamma^{-}=2 \gamma_{0}-\gamma^{+}
$$

note that (61) describes a portion of the boundary of the admissible region shown shaded in Figure 5. Using (61) in (45) to find $\dot{s}$ gives

$$
\dot{s}=c_{0} \sqrt{1-\gamma_{0}^{2}+(1 / 3)\left(\gamma^{+}-\gamma_{0}\right)^{2}}=\sqrt{\frac{1}{3} c^{2}\left(\gamma^{+}\right)+\frac{2}{3} c_{m}^{2}}<c\left(\gamma^{+}\right) .
$$

Using (61) in (44) yields the equation to determine $\gamma^{+}$:

$$
2\left(\gamma_{0}-\gamma^{+}\right) \sqrt{\frac{1}{3} c^{2}\left(\gamma^{+}\right)+\frac{2}{3} c_{m}^{2}}-\int_{\gamma^{+}}^{\gamma_{0}} c(\gamma) d \gamma=w,
$$




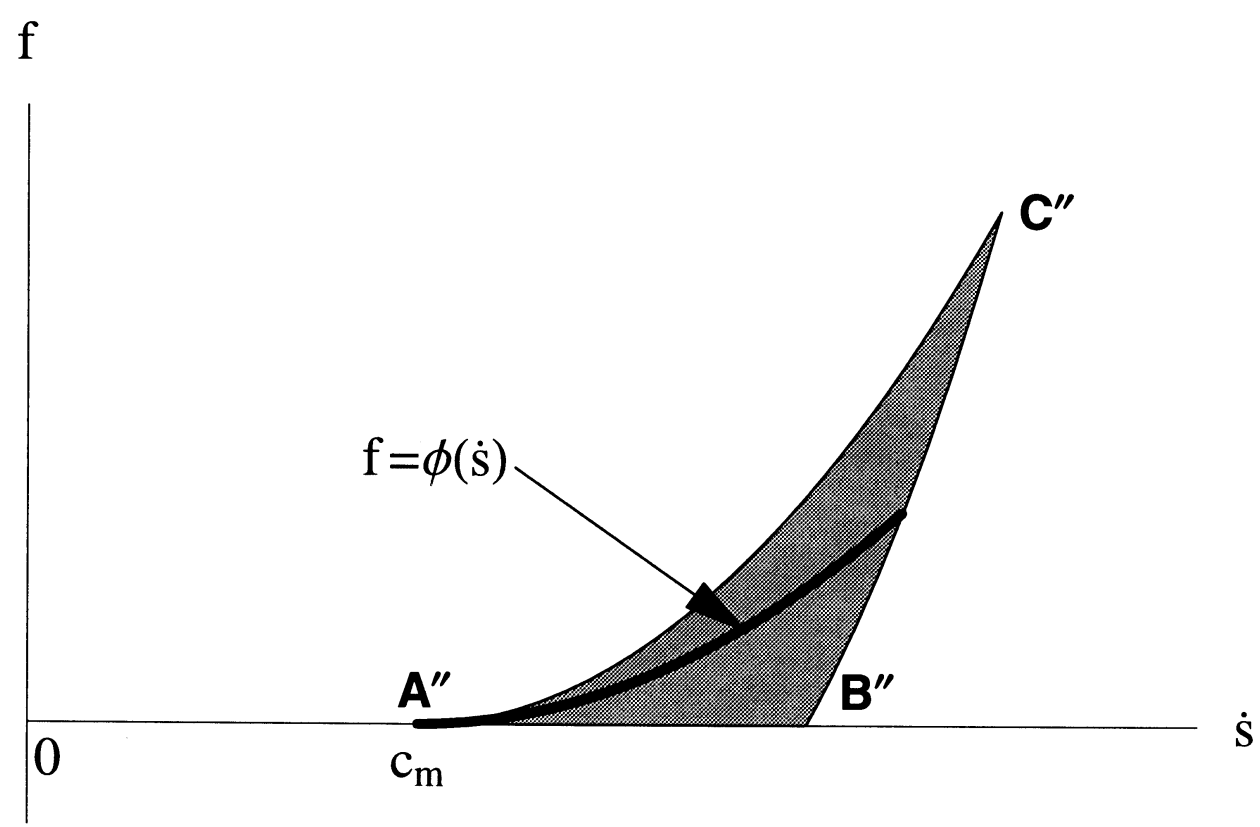

FIG. 7. The curvilinear triangle $\mathbf{A}^{\prime \prime} \mathbf{B}^{\prime \prime} \mathbf{C}^{\prime \prime}$ is the image under the mapping (17), (45) of the triangle $\mathbf{A B C}$ in the $\gamma^{+}, \gamma^{-}$-plane of Figure 5. The curve corresponding to the kinetic relation (58), shown bold, must lie in $\mathbf{A}^{\prime \prime} \mathbf{B}^{\prime \prime} \mathbf{C}^{\prime \prime}$.

where $c(\gamma)$ is given by (8) and $w$ is the departure of the impactor velocity $V$ from the critical value $V_{\star}$; see (46). By setting

$$
\gamma^{+}=\gamma_{0}-\sqrt{1-\gamma_{0}^{2}} \eta^{+}
$$

in (63) and making the corresponding change of variable in the integral, one can reduce (63) to the following problem:

Find $\eta^{+}$in $[0, h]$ such that

$$
F\left(\eta^{+}\right)=w /\left(\sqrt{1-\gamma_{0}^{2}} c_{m}\right)
$$

where $h=\gamma_{0} / \sqrt{1-\gamma_{0}^{2}}$ and $F(\eta)$ is defined by (54).

Since $F(\eta)$ is a positive, monotonically increasing function of $\eta$, (65) has a unique solution $\eta^{+}$as long as $0 \leq w /\left(\sqrt{1-\gamma_{0}^{2}} c_{m}\right) \leq F(h)$. Using the relation (46) between $w$ and $V$, we conclude that, in the case of dissipation-free kinetics, the impact problem has a unique fan-plus-shock solution provided $V$ lies in the range $V_{\star} \leq V \leq V_{\star \star}$, where $V_{\star \star}$ is given by (35). This is precisely the gap in the range of impactor velocities left unfilled by the pure-fan and pure-shock-wave solutions constructed earlier. Once $\eta^{+}$ has been determined, $\gamma^{+}=\gamma_{\text {dissfree }}^{+}(V)$ is found from $(64), \gamma^{-}=\gamma_{\text {dissfree }}^{-}(V)$ from (61), and $\dot{s}=\dot{s}_{\text {dissfree }}(V)$ from (62). The relationship $\dot{s}=\dot{s}_{\text {dissfree }}(V)$ between the shock velocity and the impactor velocity $V$ for the solution arising from dissipationfree kinetics is represented by the curve $\mathbf{A}^{\prime} \mathbf{B}^{\prime}$ in Figure 6 .

Part (b) of Figure 8 represents a schematic "photograph" of the spatial strain distribution at a fixed time instant $t$ for a value $V$ of impactor velocity in the interval 


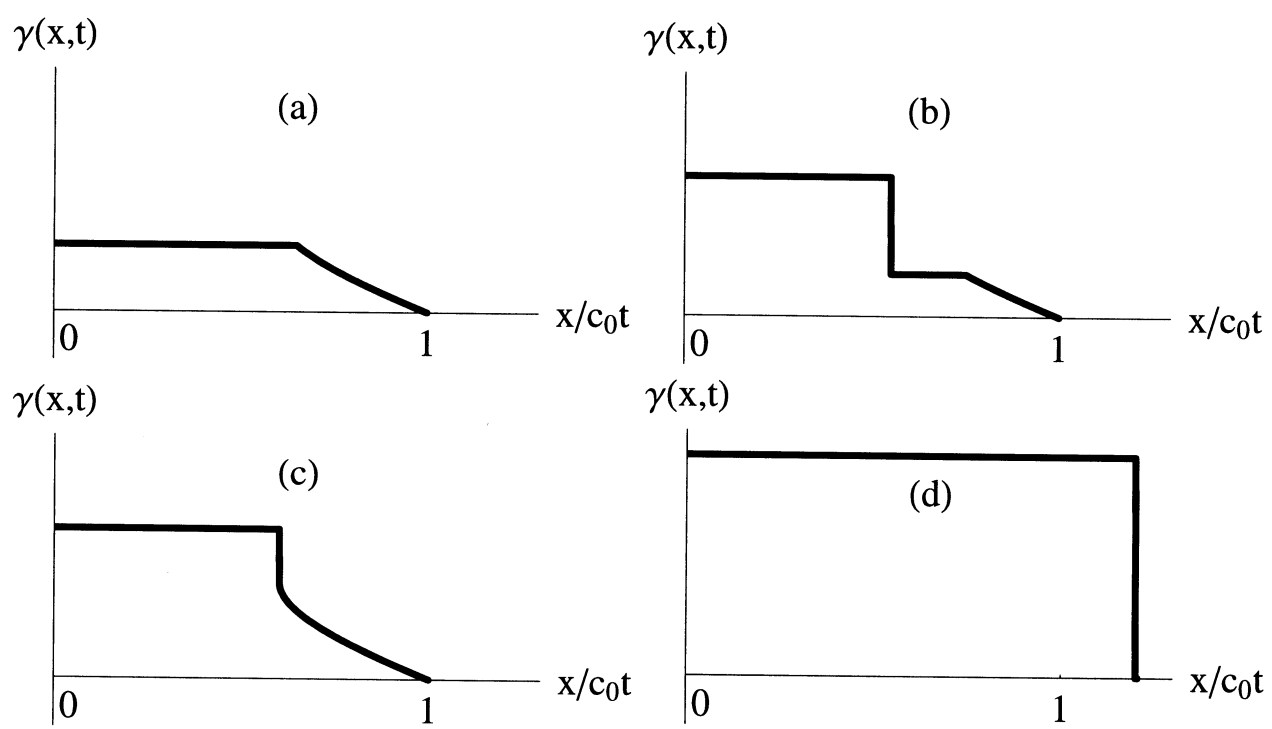

FIG. 8. Schematic plots of the strain $\gamma(x, t)$ as a function of $x$ at a fixed instant $t$ for various tensile impactor velocities $V$. Pure-fan response for a weak impact, $0<V<V_{\star}$, is shown in part (a). Parts (b) and (c) represent a two-wave, fan-plus-shock response for an impact of intermediate strength, $V_{\star}<V<V_{\star \star}$; the kinetics underlying part (b) are dissipation-free, while those for part (c) are maximally dissipative. Part (d) shows the pure-shock-wave response for a strong impact, $V>V_{\star \star \star}$.

$\left(V_{\star}, V_{\star \star}\right)$ when the mixed fan-plus-shock solution is that determined by the dissipationfree kinetic relation (60).

4.3. Maximally dissipative kinetics. We next consider a special kinetic relation that maximizes the rate of dissipation in a sense to be made clear shortly. In the context of phase-transforming materials, various notions of maximally dissipative kinetics, and their relation to other proposed selection mechanisms for nonlinear conservation laws such as the chord criterion of Oleinik [20] and Shearer [21] and the maximum entropy rate principle of Dafermos [22], have been discussed in [23, 24].

A maximally dissipative kinetic relation that is the counterpart for phasetransforming solids of the relation to be discussed here has been shown in [18] to fit the experimental data obtained by Erskine and Nellis [19] for the graphite-to-diamond transition.

Let $\gamma^{ \pm}$be the strains on either side of a discontinuity moving with a given speed $\dot{s}$. By (14), the associated dissipation rate is given by $D=f \dot{s}$, where $f=\hat{f}\left(\gamma^{+}, \gamma^{-}\right)$ is defined in (15). We ask the following question: For a given $\dot{s}$, what values of $\gamma^{+}$ and $\gamma^{-}$maximize the dissipation rate $\hat{f}\left(\gamma^{+}, \gamma^{-}\right) \dot{s}$, subject to the constraint (43)?

If one seeks necessary conditions for extrema of the dissipation rate by adjoining the constraint (43) to $\hat{f}\left(\gamma^{+}, \gamma^{-}\right) \dot{s}$ with the help of a Lagrange multiplier, one finds that

$$
\text { either } \frac{\hat{\sigma}\left(\gamma^{+}\right)-\hat{\sigma}\left(\gamma^{-}\right)}{\gamma^{+}-\gamma^{-}}=\hat{\sigma}^{\prime}\left(\gamma^{+}\right) \text {or } \quad \frac{\hat{\sigma}\left(\gamma^{+}\right)-\hat{\sigma}\left(\gamma^{-}\right)}{\gamma^{+}-\gamma^{-}}=\hat{\sigma}^{\prime}\left(\gamma^{-}\right)
$$

must hold. Thus on the stress-strain curve, the chord connecting the two states on either side of the discontinuity must be tangent to the curve at one of its end points. 
If it is now assumed that the material is characterized by the rubberlike constitutive law (5), and that $\gamma^{+}<\gamma_{0}, \gamma^{-}>\gamma_{0}$, then geometry shows that $\gamma^{+}$and $\gamma^{-}$must be disposed so that the chord lies everywhere above the stress-strain curve if the first alternative in (66) but holds everywhere below the curve for the second alternative. It follows that the driving force $\hat{f}\left(\gamma^{+}, \gamma^{-}\right)$is positive for the first alternative and negative for the second. If $\dot{s}$ is assumed to be positive, as is the case in our impact problem, then the first alternative yields positive dissipation $D$ and the second gives $D<0$. (The second alternative would be relevant for a discontinuity moving to the left so that, in contrast to the case arising here, the portion of the bar in which $\gamma<\gamma_{0}$ is growing at the expense of the portion in which $\gamma>\gamma_{0}$.) Thus for the maximum dissipation rate, we accept the first alternative in (66) and reject the second; we note that $(66)_{1}$ is equivalent to

$$
\dot{s}=c\left(\gamma^{+}\right) .
$$

In the context of the mixed fan-shock solution to the impact problem, (67) asserts that the shock wave in fact coincides with the tail of the fan, or, in other words, the fan terminates in a discontinuity when maximum dissipation rate is in force.

For the special material (5), the first alternative in (66) is readily shown to reduce to

$$
\gamma^{-}=3 \gamma_{0}-2 \gamma^{+}
$$

Note that (68) describes a portion of the boundary of the admissible region in Figure 5; cf. (61) and the remark immediately following it. If (68) is used to eliminate $\gamma^{-}$in the respective expressions (17) and (45) for driving force and shock wave speed, and if $\gamma^{+}$is then eliminated from the results, the following explicit relation between $f$ and $\dot{s}$ emerges:

$$
f=\phi(\dot{s})=\frac{9}{4} E\left(\dot{s}^{2}-c_{m}^{2}\right)^{2} / c_{0}^{4} \text { for } c_{m} \leq \dot{s} \leq c_{0}
$$

recall that $E$ is Young's modulus for the material. We call (69) the maximally dissipative kinetic relation for the rubberlike material characterized by (5).

Using (67), (68) in (44) yields the equation to determine $\gamma^{+}$:

$$
3\left(\gamma_{0}-\gamma^{+}\right) c\left(\gamma^{+}\right)-\int_{\gamma^{+}}^{\gamma_{0}} c(\gamma) d \gamma=w
$$

where $c(\gamma)$ and $w$ are given by (8) and (46), respectively. Making the change of variable (64) in (70) reduces our problem to the following:

Find $\eta^{+}$in $[0, h]$ such that

$$
G\left(\eta^{+}\right)=w /\left(\sqrt{1-\gamma_{0}^{2}} c_{m}\right),
$$

where $G(\eta)$ is defined by (56).

Since $G(\eta)$ is a positive, monotonically increasing function of $\eta$, (71) has a unique solution as long as $0 \leq w /\left(\sqrt{1-\gamma_{0}^{2}} c_{m}\right) \leq G(h)$. Using (46), we conclude that, in the case of maximally dissipative kinetics, the impact problem has a unique fan-plus-shock solution provided the impactor velocity $V$ lies in the range $V_{\star} \leq V \leq V_{\star \star \star}$, where $V_{\star \star \star}$ 
is defined in (36). Once $\eta^{+}$has been determined, $\gamma^{+}=\gamma_{\text {maxdiss }}^{+}(V)$ is found from (64), $\gamma^{-}=\gamma_{\text {maxdiss }}^{-}(V)$ from (68), and $\dot{s}=\dot{s}_{\text {maxdiss }}(V)$ from (67). The relationship $\dot{s}=\dot{s}_{\text {maxdiss }}(V)$ for the solution arising from maximally dissipative kinetics is that represented by the curve $\mathbf{A}^{\prime} \mathbf{C}^{\prime}$ in Figure 6 .

Part (c) of Figure 8 shows a schematic plot of the spatial strain distribution at a fixed time $t$ for a value of impactor velocity $V$ in the interval $\left(V_{\star}, V_{\star \star}\right)$ when the mixed fan-plus-shock solution is that determined by the maximally dissipative kinetic relation $(69)$.

4.4. Upper and lower bounds on $\gamma^{+}, \gamma^{-}$in the one-parameter family of mixed fan-shock solutions. Let us assume that, for each $V$ in $\left(V_{\star}, V_{\star \star \star \star}\right),(41)$, (42) determine $\gamma^{+}, \gamma^{-}$as smooth functions of $\dot{s}$, with $\gamma^{-}>\gamma^{+}$, along the appropriate horizontal line in the shaded region of Figure 6. Differentiating (41), (42) with respect to $\dot{s}$ with $V$ fixed provides two linear equations for $\partial \gamma^{ \pm} / \partial \dot{s}$, from which one finds that

$$
\begin{gathered}
\frac{\partial \gamma^{+}}{\partial \dot{s}}=-\frac{\left(\gamma^{-}-\gamma^{+}\right)\left\{c^{2}\left(\gamma^{-}\right)+\dot{s}^{2}\right\}}{\left\{c\left(\gamma^{+}\right)-\dot{s}\right\}\left\{c^{2}\left(\gamma^{-}\right)+\dot{s} c\left(\gamma^{+}\right)\right\}}, \\
\frac{\partial \gamma^{-}}{\partial \dot{s}}=-\frac{\left(\gamma^{-}-\gamma^{+}\right)\left\{c\left(\gamma^{+}\right)-\dot{s}\right\}}{c^{2}\left(\gamma^{-}\right)+\dot{s} c\left(\gamma^{+}\right)} .
\end{gathered}
$$

Since $\gamma^{-}>\gamma^{+}$and $\dot{s} \leq c\left(\gamma^{+}\right)$, one concludes that

$$
\frac{\partial \gamma^{+}}{\partial \dot{s}} \leq 0, \quad \frac{\partial \gamma^{-}}{\partial \dot{s}} \leq 0
$$

so that neither $\gamma^{+}$nor $\gamma^{-}$can increase as $\dot{s}$ increases with $V$ fixed. If, in particular, one has $V_{\star}<V<V_{\star \star}$, it follows from (74) and Figure 6 that $\gamma^{+}$and $\gamma^{-}$take their least values at the point on the dissipation-free $\operatorname{arc} \mathbf{A}^{\prime} \mathbf{B}^{\prime}$ determined by $V$, while their greatest values occur on the maximally dissipative $\operatorname{arc} \mathbf{A}^{\prime} \mathbf{C}^{\prime}$. Thus

$$
\left.\begin{array}{c}
\gamma_{\text {dissfree }}^{+}(V) \leq \gamma^{+}(\dot{s}, V) \leq \gamma_{\text {maxdiss }}^{+}(V), \\
\gamma_{\text {dissfree }}^{-}(V) \leq \gamma^{-}(\dot{s}, V) \leq \gamma_{\text {maxdiss }}^{-}(V)
\end{array}\right\} \text { for } V_{\star}<V \leq V_{\star \star} .
$$

Thus the special cases corresponding to maximally dissipative kinetics and dissipationfree kinetics provide upper and lower bounds, respectively, on the strains on either side of the shock for the one-parameter family of mixed fan-shock solutions arising from those impacts of intermediate strength for which $V_{\star}<V \leq V_{\star \star}$. In particular, when $V$ is in this range, the back-state strain at the shock can never exceed that arising for maximally dissipative kinetics, irrespective of the selection principle used to single out a solution from the one-parameter family.

The bounds (75) are the best possible in the sense that they are achieved for the two special kinetic relations considered in the preceding subsections.

One can use (74) in a similar way to determine bounds on $\gamma^{ \pm}$when $V$ is in the range $\left(V_{\star \star}, V_{\star \star \star}\right)$.

5. Concluding comments. In the case of models for phase-transforming elastic materials, one has $\hat{\sigma}^{\prime}(\gamma)<0$ over a finite interval of strain, with $\hat{\sigma}^{\prime}(\gamma)>0$ elsewhere, so that the system (1) is of mixed hyperbolic-elliptic type. In the special case of a trilinear two-phase material, for which the rising-falling-rising stress-strain curve is piecewise linear, an impact problem has been studied in detail in [18]. It is found that, 
as in the present case, there is a range $\left(V_{\star}, V_{\star \star \star}\right)$ of impactor velocities $V$ in which there is a one-parameter family of two-wave solutions. Each member of this family involves two moving strain discontinuities, the slower of which is a phase boundary and the faster of which is a shock wave in the untransformed material; the kinetic relation determines the phase boundary speed and hence controls the rate at which the phase transition takes place. For values of $V$ greater than $V_{\star \star \star \star}$, there is, as in the present case, a unique solution for each $V$, and it involves only one moving strain discontinuity, which is a phase boundary whose speed is determined from the data in the boundary-initial value problem alone, without supplementary kinetics. Moreover, for $V>V_{\star \star \star}$ the speed of the discontinuity is Lagrangian-supersonic with respect to undisturbed material; this too is parallel to the results found in the present case. The fact that impact may generate either a two-wave or a one-wave response, each involving a phase transition, is observed experimentally [19]; the one-wave, supersonic response for the largest impactor velocities is called the "overdriven" case in the experimental literature.

A point of difference between the present hyperbolic-but-not-genuinely-nonlinear rubberlike case and the hyperbolic-elliptic case arises for phase transitions: The nucleation criterion, necessary in the phase-transition model for the impact problem [18], is needed neither here nor in the Riemann problem for the elastodynamic system with a monotonic cubic nonlinearity [12].

Whereas a kinetic relation has a firm physical basis in the case of materials such as crystals capable of undergoing stress-induced, first-order phase transitions, the physical justification for such a relation at the molecular level for rubber is not clear. Rubber is subject to a process of crystallization, which may be generated either by cooling or by severe stretching. Moreover, such crystallization has been described as a first-order phase transition, corresponding to a definite structural change; see the discussion in sections 1.7, 1.8 of [2]. It has in the past, in fact, been proposed [25] that crystallization might be responsible for the convex portion of the tensile stress-strain curve for rubber; if this were experimentally justified, it would present a natural basis for the introduction of kinetics in the present model for the impact problem. Unfortunately, the preponderance of experimental evidence apparently does not support this view; see the discussion in section 6.9 of [2].

Quite apart from the lack of a physical basis for choosing any particular kinetic relation or regularizing augmentation to complete the model used here, there is another difficulty in attempting to force the "hyperbolic-but-not-genuinely-nonlinear" system into the mold of "hyperbolic-elliptic" phase-transition models such as that of [18]. The latter models are capable of describing equilibrium mixtures of the two material phases; nonmonotonicity of the stress-strain curve is necessary for the existence of such mixtures. This is easily seen from the jump conditions (2). Consider an equilibrium solution $\gamma, v$ of (1): $\gamma$ is independent of time, and $v=0$. Suppose $\gamma$ contains a stationary strain discontinuity $(\dot{s}=0)$. The first of the jump conditions (2) is trivially satisfied, but the second reduces to the requirement that the stress $\sigma$ must be continuous across the strain jump. This is impossible if $\hat{\sigma}(\gamma)$ is monotonic in $\gamma$ and $\gamma^{+} \neq \gamma^{-}$. Thus stress-induced equilibrium mixtures of two "phases," if phases they were, could not occur for the model arising from (5). This is consistent with the fact that, in the mixed fan-shock regime for impacts of intermediate strength, (47) requires the velocity $\dot{s}$ of the shock to be bounded away from zero $\left(\dot{s} \geq c_{m}>0\right)$; this does not occur in the models for phase transitions [18]. 
Regrettably, I have been unable to find dynamic experiments on rubber reported in the literature that might reasonably be treated using a one-dimensional tensile impact model of the kind utilized here. While there are indeed experiments involving the transient dynamic response of rubber - see, for example, $[26,27,28]$-none of these may be modeled as one-dimensional tensile impact problems.

Finally, it should be remarked that a viscoelastic model for one-dimensional purely tensile waves in a rubberlike material has been given by Mihăilescu-Suliciu and Suliciu [29].

Acknowledgment. I am indebted to a referee for calling the paper [12] by Schulze and Shearer to my attention.

\section{REFERENCES}

[1] L.R.G. Trelone, Stress-strain data for vulcanized rubber under various types of deformation, Trans. Faraday Soc., 40 (1944), pp. 59-70.

[2] L.R.G. Treloar, The Physics of Rubber Elasticity, 3rd ed., Oxford University Press, Oxford, UK, 1975

[3] R.W. Ogden, Non-Linear Elastic Deformations, Ellis Horwood Limited, John Wiley and Sons, Chichester, UK, 1984

[4] P.D. Lax, Hyperbolic Systems of Conservation Laws and the Mathematical Theory of Shock Waves, CBMS-NSF Regional Conf. Ser. in Appl. Math. 11, SIAM, Philadelphia, 1973.

[5] L.C. Evans, Partial Differential Equations, Grad. Stud. Math. 19, AMS, Providence, RI, 1998.

[6] P.G. LeFlOCH, An introduction to nonclassical shocks of systems of conservation laws, in An Introduction to Recent Developments in Theory and Numerics for Conservation Laws (Freiburg/Littenweiler, 1997), D. Kröner, M. Ohlberger, and C. Rohde, eds., Lect. Notes Comput. Sci. Eng. 5, Springer, Berlin, 1999, pp. 28-72.

[7] J.K. Knowles, Stress-induced phase transitions in elastic solids, Comput. Mech., 22 (1999), pp. 429-436.

[8] T. von KÁRmán And P. Duwez, The propagation of plastic deformation in solids, J. Appl. Phys., 21 (1950), pp. 987-994.

[9] D.A. Porter And K.E. Easterling, Phase Transformations in Metals and Alloys, Van Nostrand Reinhold, London, 1981.

[10] R. Abeyaratne And J.K. Knowles, On the driving traction acting on a surface of strain discontinuity in a continuum, J. Mech. Phys. Solids, 38 (1991), pp. 345-360.

[11] B.T. Hayes and P.G. LeFloch, Nonclassical shocks and kinetic relations: Finite difference schemes, SIAM J. Numer. Anal., 35 (1998), pp. 2169-2194.

[12] M.R. Schulze AND M.R. SheAReR, Undercompressive shocks for a system of hyperbolic conservation laws with cubic nonlinearity, J. Math. Anal. Appl., 229 (1999), pp. 344-362.

[13] R. Abeyaratne and J.K. Knowles, A continuum model of a thermoelastic solid capable of undergoing phase transitions, J. Mech. Phys. Solids, 41 (1993), pp. 541-571.

[14] L. Truskinovsky, Equilibrium phase interfaces, Soviet Physics Doklady, 30 (1982), pp. 945948.

[15] M. Slemrod, Admissibility criteria for propagating phase boundaries in a van der Waals fluid, Arch. Ration. Mech. Anal., 81 (1983), pp. 301-315.

[16] R. Abeyaratne And J.K. Knowles, Implications of viscosity and strain-gradient effects for the kinetics of propagating phase boundaries in solids, SIAM J. Appl. Math., 51 (1991), pp. $1205-1221$.

[17] P. Rosakis And J.K. Knowles, Unstable kinetic relations and the dynamics of solid-solid phase transitions, J. Mech. Phys. Solids, 43 (1997), pp. 2055-2081.

[18] R. Abeyaratne and J.K. Knowles, On a shock-induced martensitic phase transition, J. Appl. Phys., 87 (2000), pp. 1123-1134.

[19] D.J. ERskine AND W.J. NelLis, Shock-induced martensitic transformation of highly oriented graphite to diamond, J. Appl. Phys., 71 (1992), pp. 4882-4886.

[20] O.A. OleINIK, Uniqueness and stability of the generalized solution of the Cauchy problem for a quasilinear equation, Uspekhi Mat. Nauk (N.S.), 14 (1959), pp. 165-170.

[21] M. Shearer, The Riemann problem for a class of conservation laws of mixed type, J. Differential Equations, 46 (1982), pp. 426-443. 
[22] C.M. Dafermos, Hyperbolic systems of conservation laws, in Systems of Nonlinear Partial Differential Equations, J.M. Ball, ed., D. Reidel, Dordrecht, 1983, pp. 25-70.

[23] R. Abeyaratne and J.K. Knowles, On the propagation of maximally dissipative phase boundaries in solids, Quart. Appl. Math., 50 (1992), pp. 149-172.

[24] R. Abeyaratne and J.K. Knowles, Nucleation, kinetics and admissibility criteria for propagating phase boundaries, in Shock-Induced Transitions and Phase Structures in General Media, R. Fosdick, E. Dunn, and M. Slemrod, eds., IMA Vol. Math. Appl. 52, SpringerVerlag, New York, 1992, pp. 1-33.

[25] P.J. FLORY, Thermodynamics of crystallization in high polymers. I. Crystallization induced by stretching, J. Chemical Physics, 15 (1947), pp. 397-408.

[26] P. MAson, Finite elastic wave propagation in rubber, Proc. Roy. Soc. London Ser. A, 272 (1963), pp. 315-330.

[27] H. Kolsky, Production of tensile shock waves in stretched natural rubber, Nature, 224 (1969), p. 1301.

[28] J.L. Wegner and J.B. Haddow, An experimental study of the unloading waves in a plucked hyperelastic string, J. Appl. Mech., 57 (1990), pp. 667-671.

[29] M. MinĂilescu-Suliciu And I. Suliciu, On tensile shock waves in rubber-like materials, J. Appl. Mech., 54 (1987), pp. 498-502. 